\title{
Surface and bulk characterization of molten In and In-Sn alloys
}

\author{
F. Gauzzi ${ }^{1}$,D. Giuranno ${ }^{2}$, R. Montanari ${ }^{1}$, R. Novakovic ${ }^{2}$, E. Ricci ${ }^{2}$ and A. Varone ${ }^{1}$ \\ ${ }^{1}$ Dipartimento di Ingegneria Meccanica- Università di Roma-Tor Vergata,Via del Politecnico,1 00133 Roma Italy \\ ${ }^{2}$ CNR- IENI Istituto per l'Energetica e le Interfasi,Via De Marini, 616149 Genova Italy
}

\begin{abstract}
In this work a double contribution to the characterization of molten In and In-Sn alloys considered as main components of an important class of lead free solder materials is shown: the study of the influence of oxygen on the capillary phenomena and the XRD investigation of the structure of liquid in a range of temperatures around that of liquidus. The surface tension behaviour of In-Sn binary alloys at different compositions, in terms of effective oxygen pressure, were compared with the data of pure In and the theoretical predictions, revealing that the lower oxidizability of indium was shown to control indium-tin alloys with a tin content up to about 80 at $\%$, due to the presence of the most volatile oxide In2O. From the XRD spectra the radial distribution functions (RDF) have been determined for each alloys. Experiments of High Temperature X-ray diffraction (HT-XRD) showed that atomic clustering forms in the melt immediately before the appearing of the first solid. The structure of clusters is correlated to that of solid.
\end{abstract}

\section{Introduction}

The In-Sn based alloys are principally used as lead free brazing materials $[1,2]$ due to unique combination of their properties as well as a good alloying tendency with copper, widely used as substrate in electronic industry [3]. They are also used as constituents of complex gold brazers as well as of dental alloys [4]. The In-Sn system has been proposed for high reliability laser die material attachment and bonding to heat sinks. Although many authors have studied the thermodynamic and the thermophysical properties of In-Sn liquid alloys over the wide range of composition and temperature, some contradictions concerning the structure of the melts still exist [5-8].

Besides, since the solder process is determined by the physico-chemical properties of the liquid solder/solid substrate system and by the environmental conditions over which the solder process is carried out, a characterisation of the surface properties (surface tension, surface segregation) as well as of the wetting behaviour is needed. In particular, surface oxidation can drastically change the surface tension with a strong effect on the way the liquid metal wets the substrate.

This work reports preliminary results of an experimental campaign aimed to find possible correlation between surface and bulk properties of liquid metals. Accordingly, a double contribution to the characterization of molten In and In-Sn alloys is here presented:

- Dynamic Surface Tension (DST) measurements addressed to validate the theoretical approach able to explain the mechanisms that keep the surface of molten metallic system oxygen-free despite the thermodynamic driving force. These mechanisms involve, in particular, the evaporation of metal oxides from the metal interface and make it possible to define the effective oxygen partial pressure consistent with the saturation of a steady liquid phase [9]. Experimentally, the surface tension is utilised as the sensitive parameter for the interactions between the oxygen and the liquid metal surface, revealed by a change in the sign of the surface tension temperature coefficient. If enough time is not allowed for the system to reach equilibrium or steady state, the measurements are done under dynamic conditions and the measured parameter is the DST [10]. The experimental findings by DST measurements of In and In-Sn alloys, here shown, substantially confirm the theoretical predictions. From a thermodynamic point of view, indium should be more oxidizable than tin, while, on the contrary, the volatility of the oxides favour indium, so that indium maintains the cleanness of the surface under larger oxygen partial pressures; in other words indium is, in practice, less oxidizable than tin. In fact, the theory [10-11] indicated that the reaction of the alloy to oxidation tends to be similar to that of the less-oxidizable component thanks to the contribution of the volatile oxide which should prevent the surface oxidation even if the corresponding metal is present in low percentages.

- High Temperature X-ray diffraction (HT-XRD) permits the direct monitoring of phase transformations [15-18] and present experiments have been addressed to investigate possible structural changes occurring in the 
melt just before the formation of the first solid and during the solidification.

Pure In and two binary alloys, In90-Sn10 and In50-Sn50, have been investigated. The alloy compositions have been suitably chosen to study both the peritectic reaction (In90-Sn10) and the eutectic reaction (In50-Sn50).

Melts have been slowly cooled down step by step till complete solidification, XRD patterns were recorded at constant temperature and the related radial distribution functions (RDF) calculated. The results were examined by focusing the attention on atomic clustering in the liquid phase correlated to the solid crystalline structure.

\section{Materials and experimental}

The examined materials are different In-Sn alloys; the bars in figure 1 indicate the compositions investigated by DST and HT-XRD.

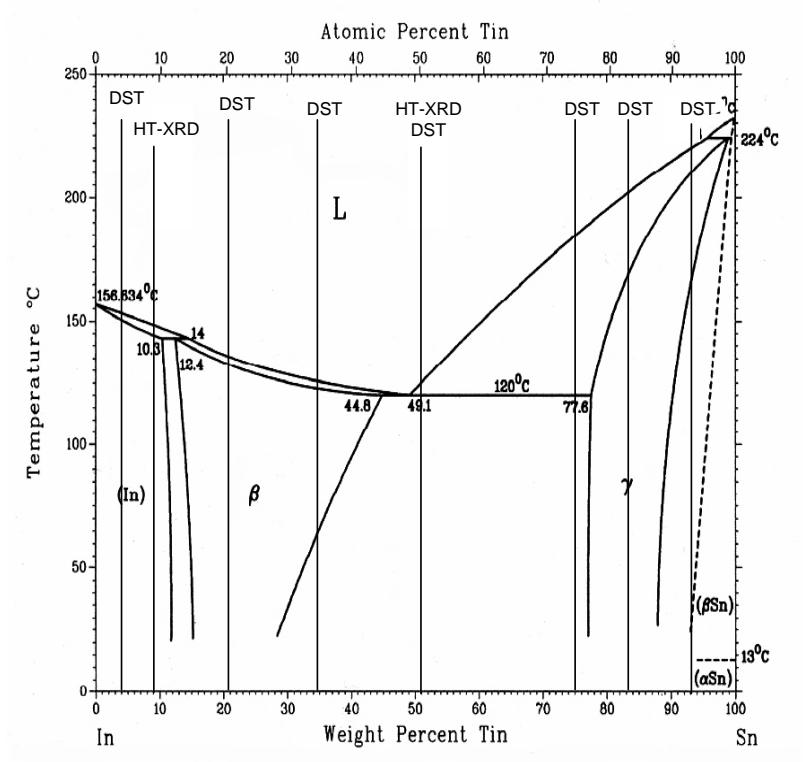

Fig. 1. In-Sn equilibrium phase diagram and the compositions of the investigated alloys [ 19 ].

The surface tension measurements of molten Indium and In-Sn alloys were performed by the large-drop method under dynamic conditions (i.e. varying the temperature at constant oxygen partial pressure). The alloy specimens were prepared by mixing high purity Indium and Tin (99.9999\% Marz-grade) melted under a vacuum of $10^{-4}$ $\mathrm{Pa}$ at $723 \mathrm{~K}$. Each nominal composition was controlled by SEM/EDS analyses. Samples of about $2.5 \mathrm{~g}$ were prepared and then mechanically abraded and chemically cleaned with an organic solvent in an ultrasonic bath. The sample was placed in a non-oriented monocrystalline alumina (sapphire) crucible $(\mathrm{r}=5.5 \mathrm{~mm})$. The crucible with the sample was laid on an alumina holder sliding in the experimental apparatus which is described in detail elsewhere [12-14]. When the apparatus conditions (temperature, oxygen partial pressure) were reached, the sample was introduced into the centre of the furnace by a pushrod magnetic manipulator. All measurements were performed under a vacuum $\approx 10^{-4} \mathrm{~Pa}$ and an oxygen content $\mathrm{P}_{\mathrm{O} 2} \leq 10^{-4} \mathrm{~Pa}$, monitored by a solid state electrode, placed close to the metal sample [10]. The surface tension measurements were carried out in the temperature range between 473 and $823 \mathrm{~K}$ by decreasing and increasing temperature at a rate $5 \mathrm{~K} / \mathrm{min}$ The liquid metal drop profile was acquired by a CCD camera and processed with ad hoc acquisition software (A.S.T.R.A.view). This acquisition procedure allows real time surface tension measurements to be performed with an accuracy of $\pm 0.1 \%$ and at the same time the values of the other parameters (temperatures, oxygen partial pressure) to be followed. Due to its high performance in terms of time of acquisition and reliability (up to 10 points per second with an accuracy up to $0.1 \%$ ), it is particularly suitable for the dynamic measurements. The magnification factor of the system was calculated for each acquisition with an internal reference. A detailed description of the experimental procedure is reported in [10].

HT-XRD measurements have been carried out by means of an ANTON PAAR HT-16 camera with a sample holder apt to contain molten metals and the atmosphere of inert gas (argon) avoided metal oxidation.

Melts have been cooled down through the liquidus temperature to reach a complete solidification. XRD spectra were recorded at decreasing temperatures with steps of $1 \mathrm{~K}$ for monitoring the structural changes occurring in the melt before the formation of the first solid and during the liquid-solid transformation. Temperature was kept constant while XRD data were collected.

Mo-K $\alpha$ radiation $(\lambda=0.07093 \mathrm{~nm})$ has been employed in XRD measurements; diffraction patterns were collected in the $2 \Theta$ angular range $5-55^{\circ}$ with steps of $0.05^{\circ}$ and counting time of $5 \mathrm{~s}$ for each step. From each XRD pattern the radial distribution function (RDF) has been then determined; the method of analysis was described elsewhere [20].

\section{Results and discussion}

\subsection{DST}

In order to highlight the interactions between the oxygen and the liquid metal surface, the behaviour of the surface tension over the time is observed. An isobaric procedure is adopted, where the imposed temperature is changed under the constant oxygen partial pressure of the feed gas until the tensioactive effect is obtained, as revealed by the change of the sign of the temperature coefficient. As saturation conditions strongly depend on temperature, a temperature modulation corresponds to a modulation in the saturation level and is then equivalent to a modulation in the oxygen concentration in the liquid phase. The existence of the effective oxygen pressure, which is well above the saturation equilibrium curve, has been demonstrated [12-14]. In figure 2 the results of a DST test of pure In, performed under isobaric conditions, varying the temperature at a rate $5 \mathrm{~K} / \mathrm{min}$ is shown. The temperature modulation made it possible to observe some 
significant inversions in the behaviour of the surface tension. The meaning of the inversions is clearly explained in $[10,12]$ : if a negative temperature coefficient is considered as a characteristic of a pure metal-system, the oxygen tensioactive effect over the liquid becomes evident when, with a decrease in temperature, the slope of the surface tension becomes positive. Then, the temperature coefficient becomes negative after the appearance of a second inversion point, at which point the oxygen effect disappears and the surface of the sample reaches again its clean state. The position of the inversion points are approximately symmetrical, as a sign of the reversible oxygen dissolution in the metal drop. The portion of the curve between the two inversions reflects the surface adsorption due to the segregation of the oxygen dissolved into the liquid bulk (a consequence of the variation of the temperature) more than the effect of the externally-imposed oxygen partial pressure. The experimental conditions of the inversion points (coordinates) are linked to tension-active effect, but not necessarily to the formation of stable oxides on the surface and this effect is identified by the sign inversion of the temperature coefficient of the surface tension.

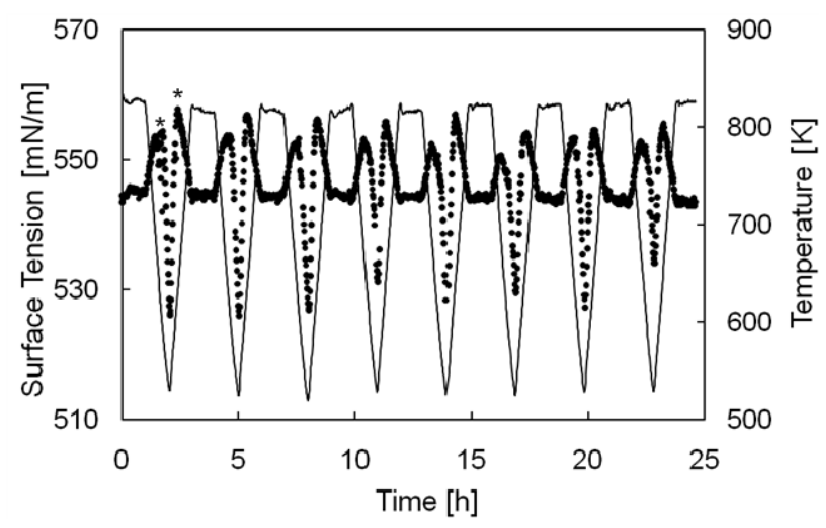

Fig. 2. Surface tension of Indium as a function of time under Knudsen regime $\left(\mathrm{P}_{\text {tot }} \approx 10^{-4}\right)$ at constant oxygen partial pressure $\left(\mathrm{P}_{\mathrm{O} 2}=2.610^{-4} \mathrm{~Pa}\right)$. The temperature was increased and decreased between 533 and $833 \mathrm{~K}$; * inversion points.

Examples of DST measurements of the In-Sn alloys are shown in Fig. 3a and 3b. The alloys investigated were In80-Sn20 and In50-Sn50 respectively. The tests were performed under the Knudsen regime $\left(\mathrm{P}_{\text {tot }} \approx 4 \cdot 10^{-4} \mathrm{~Pa}\right)$ in isobaric conditions $\left(\mathrm{P}_{\mathrm{O} 2} \approx 10^{-5} \mathrm{~Pa}\right)$. The variation in the temperature over time was set at $5 \mathrm{~K} / \mathrm{min}$, the temperature was in the ranges $465-765 \mathrm{~K}$ and $530-830 \mathrm{~K}$ respectively. In particular, in figure $3 \mathrm{a}$, a sequence of inversion points occurring in the first twelve hours that progressively become less evident was observed as well as an increase in the value of the surface tension corresponding to the lowest temperature value. This behaviour was not observed for the In50-Sn50 alloy (figure 3b). However, as already discussed in $[10,12]$, the trend reported in the figures is not correlated to the value of $\mathrm{P}_{\mathrm{O} 2}$ inside the test chamber, but is linked to the initial concentration of the dissolved oxygen in the metal sample.
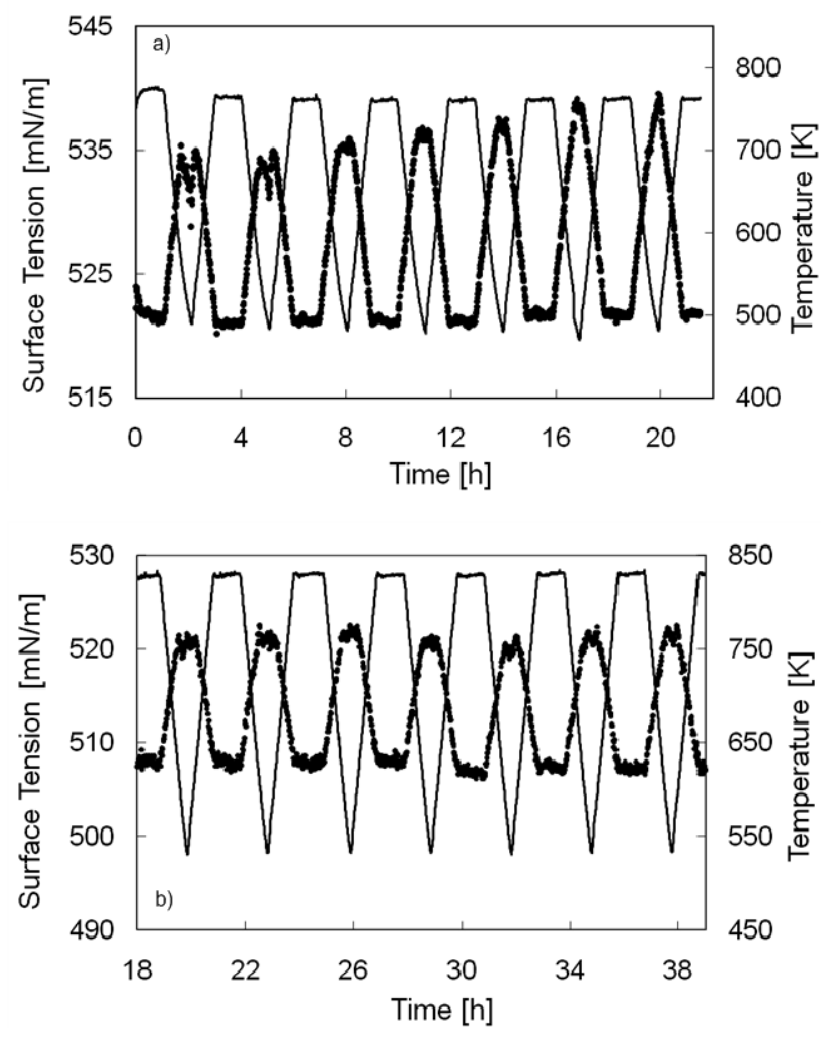

Fig. 3. Surface tension of In-Sn alloys as a function of temperature and time a) In80-Sn20 ; b) In50-Sn50.

According to our model [11], the effective oxygen pressure of the indium-tin alloy should be experimentally indistinguishable from that of pure indium up to a tin content of about 80 at $\%$. The formation of $\operatorname{In}_{2} \mathrm{O}$ prevails over the $\mathrm{SnO}$ and the lower oxidizability of indium was shown to control the behaviour of indium-tin alloys. The theoretical predictions of the effects of both temperature and composition have to be compared with the experimental findings. From the coordinates of the inversion points observed during the DST measurements performed at $750 \mathrm{~K}$, the experimental evidences of oxidation-deoxidation transitions are shown. In figure 4 these experimental data are shown as a function of the alloy composition and compared with the calculated effective oxygen pressure, which theoretically predicts the oxidation-deoxidation transition of the In-Sn system. The comparison highlights that the effective oxygen pressure must be considered as a limit property: no oxide phase can form when $\mathrm{P}_{\mathrm{O} 2}<\mathrm{P}_{\mathrm{O} 2 \mathrm{eff}}$, but the oxide phase may also be absent at $\mathrm{P}_{\mathrm{O} 2}>\mathrm{P}_{\mathrm{O} 2 \mathrm{eff}}$, on the condition that some other mechanisms, different from gas-phase such as the nucleation of the oxide phase, segregation at the interface (i.e. the non-ideal liquid phase) or oxide-fog formation inside the gas phase. Another important point is that the experimental findings do not contradict the substantial invariance in the oxidizability of indium-tin alloys with a tin content ranging from 0 to about 80 at $\%$, as evidenced by the vertical line drawn in the graph of figure 4. 


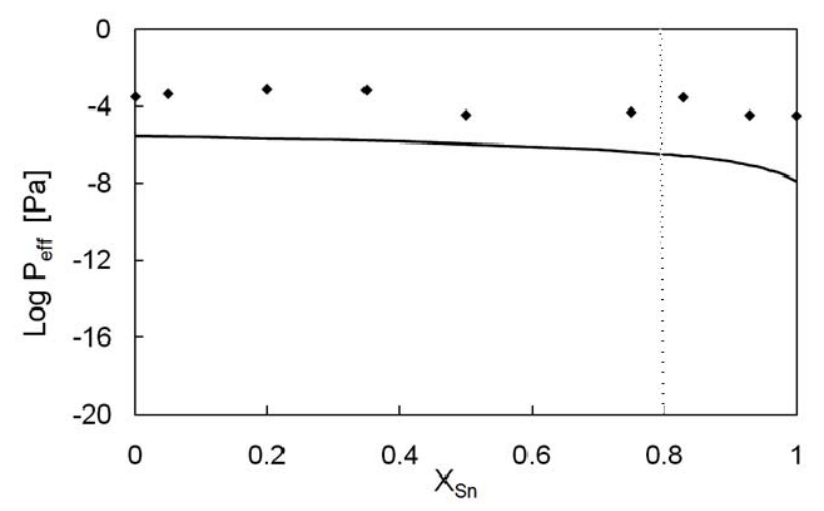

Fig. 4. The effective oxygen pressure of indium-tin alloys at a constant temperature $(\mathrm{T}=750 \mathrm{~K})$ as a function of alloy composition. ( ) Experimental values; Bold line: calculated effective oxygen pressure.

\subsection{HT-XRD}

Figures 5 and 6 display some XRD patterns of the two alloys recorded at decreasing temperatures. In figure 5 (In90-Sn10 alloy) the spectra recorded above $417 \mathrm{~K}$ exhibit the typical diffraction patterns of liquids. The spectrum at $417 \mathrm{~K}$, together with those collected at lower temperatures, shows also the $\{103\}$ and $\{321\}$ reflections of crystalline indium.

In the case of In50-Sn50 alloy the formation of the first solid (see spectrum at $395 \mathrm{~K}$ in figure 6) involves the appearing of the $\{110\}$ reflection of the hexagonal $\gamma$ phase. As temperature reaches the eutectic temperature of $393 \mathrm{~K}$ the reflections of the tetragonal $\beta$ phase are also observed in the diffraction pattern.

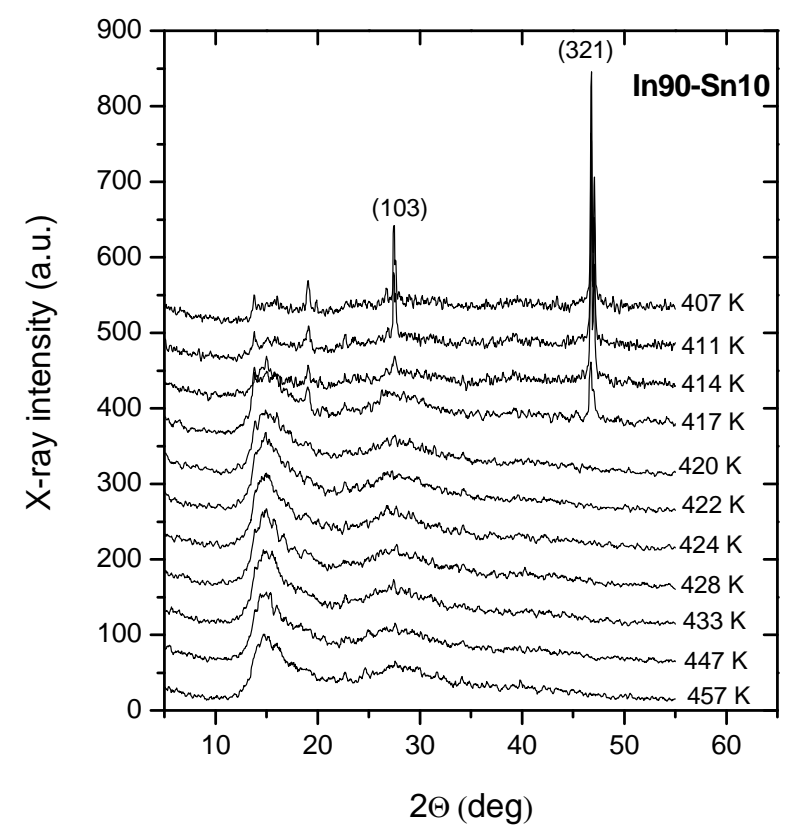

Fig. 5. XRD patterns of the In90-Sn10 alloy collected at decreasing temperatures.

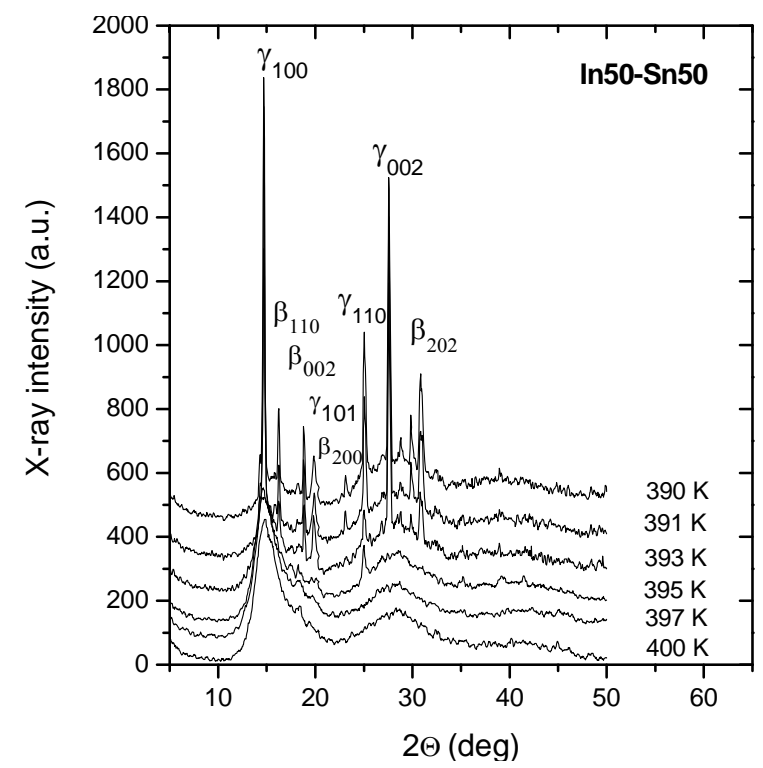

Fig. 6. XRD patterns of the In50-Sn50 alloy collected at decreasing temperatures.

For a poli-atomic liquid the curve $\sum_{U C} K_{m} \cdot 4 \pi r^{2} \cdot \rho_{m}(r)$ represents the superposition of radial distribution functions for each kind of atoms in the unit of composition (UC). $K_{m}$ is the average effective electron number for atoms of type $m$. It is defined differential RDF the same function after subtraction of the average electron density in the sample, i.e.

$\sum_{U C} K_{m} \cdot 4 \pi r^{2} \cdot\left[\rho_{m}(r)-\rho_{e}(r)\right]$

The differential RDFs calculated from patterns in figure 5 and figure 6 are displayed in figure 7 and figure 8 , respectively. The method to determine the RDF from XRD pattern was described elsewhere [20].

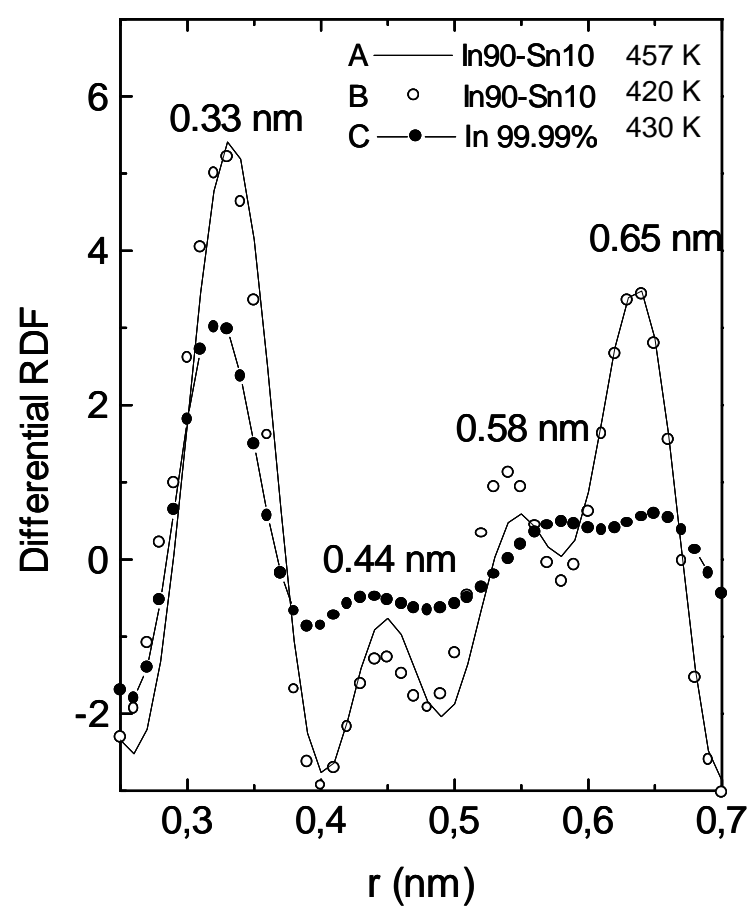

Fig. 7. Differential RDF curves of In90-Sn10 alloy at $457 \mathrm{~K}$ (A) and $420 \mathrm{~K}(\mathrm{~B})$, of In at $430 \mathrm{~K}(\mathrm{C})$. 
In figure 7 the differential RDF curves of In90-Sn10 alloy recorded at $457 \mathrm{~K}(\mathrm{~A})$ and $420 \mathrm{~K}$ (B) are compared with that of indium $(\mathrm{C})$ at the melting point $(430 \mathrm{~K})$. As shown by the In-Sn phase diagram (figure 1), $420 \mathrm{~K}$ for In90-Sn10 is the temperature just above that of liquidus. The first peak is a little broader in A and B than in C because it is the overlapping of the Sn-Sn and In-In 1st nearest neighbours components, centred at $0.32 \mathrm{~nm}$ and $0.33 \mathrm{~nm}$ respectively. The two components are too close to be distinguished. The two peaks at $0.58 \mathrm{~nm}$ and 0.65 $\mathrm{nm}$ are due to the $2 \mathrm{nd}$ nearest neighbours In-In and Sn-Sn [21-22].

Both the curves A and B of In90-Sn10 alloy exhibit the same peaks of curve $\mathrm{C}$ even if they are more pronounced and a little shifted. In addition, curves $\mathrm{A}$ and $\mathrm{B}$ show a subsidiary peak at $0.44 \mathrm{~nm}$ due to In-Sn nearest neighbours, which is close to the atomic distance between 3 rd and 4th neighbours in $\beta$ phase (see Table 1). This peak is of negligible intensity in the curve of indium (C). The intensity of the peak at $0.44 \mathrm{~nm}$ becomes lower when the temperature decreases and approaches the liquidus boundary indicating that indium and tin atoms start to separate in the melt favouring the formation of the In-rich solid phase.

Figure 8 displays the differential RDF curves of In50Sn50 alloy recorded at different temperatures. As shown by the In-Sn phase diagram (figure 1), the first solid start to form at $395 \mathrm{~K}$.

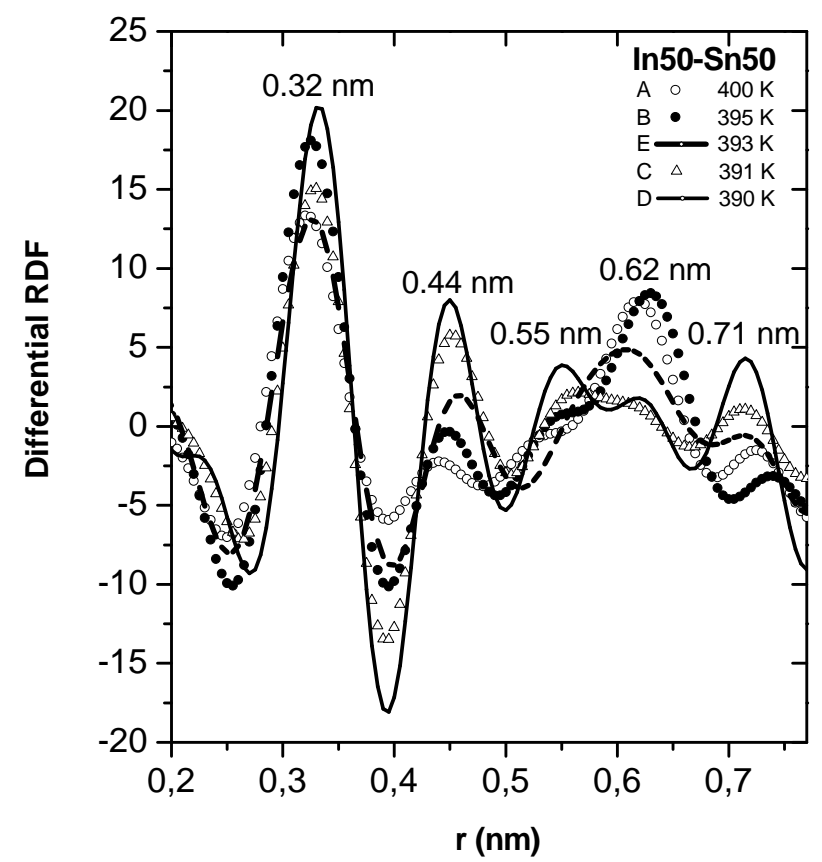

Fig. 8. Differential RDF curves of $\operatorname{In} 50-\operatorname{Sn} 50$ alloy at decreasing temperatures.

The peaks due to the overlapping of 1st and 2nd Sn-Sn and In-In nearest neighbours components are a little shifted towards lower distances with respect those of In90-Sn10 alloy. The small shift, very close to the experimental error, can be ascribed to the different compositions of the examined alloys.

After the formation of the first solid ( $\gamma$ phase) the intensities of the RDF peaks at $0.44 \mathrm{~nm}$ and $0.71 \mathrm{~nm}$ progressively increase. This variation precedes and accompanies the formation of the second solid phase, the tetragonal $\beta$ phase. The space group of $\beta$ phase is still controversial [24-25]; the body centred tetragonal lattice described in [24] is in very good agreement with XRD spectra of this material thus this model has been adopted for calculating the distances of nearest neighbours reported in Table 1. If one considers these distances it is clear that there is a strict correspondence between the atomic distances in the melt and in the solid. In other words, the number of atomic clusters with distances close to those of nearest neighbours in the $\beta$ phase raises in the melt before the formation of this crystalline phase and accompanies the transformation. Therefore, the progressively higher intensities of the RDF peaks at 0.44 $\mathrm{nm}$ and $0.71 \mathrm{~nm}$ as the eutectic temperature is approached can be considered as an indication of the increasing number of $\beta$ phase clusters.

A similar phenomenon occurs also when the $\gamma$ phase forms even if it is less evident because the distances of the nearest neighbours are very close to that of the major RDF peak. However, before the solidification of the $\gamma$ phase a new peak appears at $0.55 \mathrm{~nm}$ and becomes more intense as solidification proceeds.

Table 1- Nearest neighbours distances in the $\gamma$ and $\beta$ phases of the crystalline In50-Sn50 alloy.

\begin{tabular}{|c|c|c|}
\hline Nearest neighbors & $\begin{array}{c}\text { Distance } \\
\text { (nm) }\end{array}$ & Phase \\
\hline 1st & 0.299 & $\gamma$ (hexagonal) [23] \\
2nd & 0.321 & $\mathrm{a}=0.321 \mathrm{~nm}$ \\
3rd & 0.438 & $\mathrm{c}=0.299 \mathrm{~nm}$ \\
4th & 0.556 & \\
5th & 0.631 & \\
\hline 1st & 0.329 & $\beta$ (body centred \\
2nd & 0.346 & tetragonal) [24] \\
3rd & 0.439 & $\mathrm{a}=0.346 \mathrm{~nm}$ \\
4th & 0.489 & $\mathrm{c}=0.439 \mathrm{~nm}$ \\
5th & 0.559 & \\
6th & 0.589 & \\
6th & 0.657 & \\
8th & 0.702 & \\
\hline
\end{tabular}




\section{Conclusions}

In this work a double contribution to the characterization of molten In and In-Sn alloys is presented:

- From the XRD spectra the radial distribution functions (RDF) have been determined for each alloys. Experiments of High Temperature X-ray diffraction (HTXRD) showed that atomic clustering forms in the melt immediately before the appearing of the first solid with distances close to those of nearest neighbours in the solid phase.

- The DST experimental data in terms of effective oxygen pressure of In-Sn binary alloys of different compositions were compared with the data for the pure In and the theoretical predictions, revealing that the lower oxidizability of indium controls the indium-tin alloys with a tin content up to about $80 \mathrm{at} \%$, due to the presence of the most volatile oxide In $2 \mathrm{O}$.

\section{References}

[1] J.W. Morris Jr., J.L. Freer Goldstein, Z. Mei, JOM 45 (1993) 25.

[2] N. Moelans, K.C. Hari Kumar, P. Wollants, J. of Alloys and Compounds 360 (2003) 98.

[3] F. Gnecco, E. Ricci, S. Amore, D. Giuranno, G. Borzone, G. Zanicchi, R. Novakovic, J. Adhes. Adhesiv. 27 (2007) 409.

[4] W.S.Rapson,T. Groenwald, Gold Usage, Academic Press Inc., New York, (1978).

[5] F.-Q. Zu, X.-F. Li, L.-J. Guo, H. Yang, X.-B. Qin, Z.-G. Zhu, Physics Letters A 324 (2004) 472.

[6] C. Sun, H. Geng, Z. Yang, J. Zhang, R. Wang, Materials Characterization 55 (2005) 383.

[7] A.Q. Wu, L.J. Guo, C.S. Liu, E.G. Jia, Z.G. Zhu, Physica B 392 (2007) 323.
[8] I. Kaban, S. Gruner, W. Hoyer, A. Il'inskii, A. Shpak, J. of Non-Crystalline Solids, 353 (2007) 1979.

[9] E. Arato, E. Ricci, P. Costa, J Mater Sci. 40 (2005) 2133.

[10] E. Ricci, E. Arato, A. Passerone, P. Costa, Adv Colloid Interface Sci. 117 (2005) 15.

[11] E. Arato, E. Ricci, P. Costa, Surf. Sci. 602/1 (2008) 349.

[12] L. Fiori, E. Ricci, E. Arato, Acta Mater. 51 (2003) 2873.

[13] E. Ricci,T. Lanata, D. Giuranno, E. Arato, J. Mater Sci. 43 (2008) 2971.

[14] E. Ricci, D. Giuranno, E. Arato, P. Costa, Materials Science and Engineering A, 495 (2008) 27.

[15] P. Gondi, R. Montanari, E. Evangelista and G. Buroni, Microgravity Quarterly,7-4 (1998) 155.

[16] R. Montanari and G. Costanza, Proc. of the 1st International Symposium on Microgravity Research and Applications in Physical Sciences and Technologies, Sorrento (2000), 417.

[17] G. Costanza, F. Gauzzi and R. Montanari, Annals of the New York Academy of Sciences, 74 (2002) 67.

[18] S.I. Mudryi, T.I. Lutsishin, A.V. Korolyshin, Inorganic Materials, 40-n 12, (2004) 1284.

[19] T. B. Massalski, Binary Alloy Phase Diagram 2, American Society for Metals (1987) 1401.

[20] R. Montanari, F.Gauzzi, Annals of the New York Academy of Sciences, 1161 (2009) 407.

[21] R. Montanari, Int. J. of Mater. \& Prod. Tech., 20, Nos. 5/6 (2004) 452.

[22] H. Richter, Proc. of the Symposium on liquids: Structure, Properties, Solid interactions, General motors research laboratories, Warren-USA (1963).

[23] JCPDS-International Centre for Diffraction Data 7396.

[24] V. Bhattacharya, K. Chattopadhyay, Material Science and Engineering A, 375-377 (2004) 932.

[25] R.M. Screaton, Acta Crystallographica, 7 (1954) 364. 